\author{
United Nations Educational Scientific and Cultural Organization \\ and \\ International Atomic Energy Agency \\ THE ABDUS SALAM INTERNATIONAL CENTRE FOR THEORETICAL PHYSICS
}

\title{
MACH CONES IN SPACE AND LABORATORY DUSTY MAGNETOPLASMAS
}

\author{
A.A. Mamun $^{1}$ \\ Department of Physics, Jahangirnagar University, Savar, Dhaka, Bangladesh \\ and \\ The Abdus Salam International Centre for Theoretical Physics, Trieste, Italy \\ and \\ P.K. Shukla \\ Institut für Theoretische Physik IV, Ruhr-Universität Bochum, \\ D-44780 Bochum, Germany \\ and \\ The Abdus Salam International Centre for Theoretical Physics, Trieste, Italy.
}

\begin{abstract}
We present a rigorous theoretical investigation on the possibility for the formation of Mach cones in both space and laboratory dusty magnetoplasmas. We find the parametric regimes for which different types of Mach cones, such as dust-acoustic Mach cones, dust magneto-acoustic Mach cones, oscillonic Mach cones, etc. are formed in space and laboratory dusty magnetoplasmas. We also identify the basic features of such different classes of Mach cones (viz. dust-acoustic, dust magneto-acoustic, oscillonic Mach cones, etc.), and clearly explain how they are relevant to space and laboratory dusty manetoplasmas.

MIRAMARE - TRIESTE
\end{abstract}

July 2004

\footnotetext{
${ }^{1}$ Regular Associate of the Abdus Salam ICTP.
} 


\section{Introduction}

It is well known that an object moving with a supersonic speed in a dispersive medium creates a pressure disturbance that is not felt upstream from the object. The cone that confines this disturbance is called a Mach cone. The formation of Mach cones is well known in gases [1, 2]. These are produced, for examples, by bullets and supersonic jet planes in gases. The disturbances produced by ships in water, which are formed behind ships in deep water [3], have shapes similar to Mach cones. Mach cones also occur in solids/crystals [4, 5, 6, 7, 8, 9]. The occurrence of Mach cones in rocks is also an interesting seismological phenomenon in which sound waves travelling in a liquid-filled bore hole moves faster than the sound speed in the surrounding rocks [10].

The structure and dynamics of Saturn's rings continue to present surprises and challenges to celestial mechanics. The first surprise came in October 1980 when Voyager 1 sped past the Saturn and sent back lots of information and images, including pictures of mysterious dark spokes [11] sweeping around Saturn's B-ring. The observations from Voyager 1 revealed that the structure and dynamics of Saturn's rings are mainly dominated by charged dust particles [12]. The CASSINI spacecraft, which is now orbiting around Saturn, promises to yield even more detailed information on Saturn's rings. Since a direct probing of Saturn's dense rings is not practically possible due to the danger of collisions, we must resort to remote sensing to investigate the physical conditions within such dense rings $[13,14,15]$. We can do this by the stellar occultation measurements, or through the observations of absorption of high-energy particles or radio-waves or light scattering by dust particles at different angles. It has been proposed that observations of Mach cones in dusty plasmas might play an interesting role as a potential diagnosis method, since they can be directly viewed from outside Saturn's dusty rings, and can be used for deducing information regarding the physical state of the ambient dusty plasma $[13,14,15]$.

Havnes et al. $[13,14]$ theoretically predicted the existence of dust-acoustic (DA) Mach cones in Saturn's dusty rings. They employed the phase speed of the DA waves (DAWs [16]) for an unmagnetized dusty plasmas to obtain the Mach cone opening angle. However, there are two conditions to be satisfied for the formation of Mach cones by the DAWs in Saturn's rings. These are: i) the DAWs must propagate in the equatorial plane (in a plane perpendicular to the magnetic field) in which a dust boulder moves [17], i.e. $k_{\perp} \gg k_{z}$, where $k_{\perp}\left(k_{z}\right)$ is the DA wave-vector perpendicular (parallel) to the magnetic field, and ii) the dust boulder speed $V_{d}$ must be larger than the DA wave phase speed $V_{p}$, i.e. $V_{d} / V_{p}>1$. For Saturnian ring plasma parameters $\left(B_{0} \simeq 0.2 \mathrm{G}\right.$ and $\left.T_{i} \simeq 10 \mathrm{eV}\right)$ the ion gyro-radius $\rho_{i}\left(=V_{T i} / \omega_{c i}\right)$ is of the order of 25 $\mathrm{m}$, where $V_{T i}=\left(T_{i} / m_{i}\right)^{1 / 2}$ is the ion thermal speed, $\omega_{c i}=e B_{0} / m_{i} c$ is the ion gyro-frequency, $T_{i}$ is the ion temperature in units of the Boltzmann constant, $B_{0}$ is the magnitude of the magnetic field strength $\mathbf{B}_{\mathbf{0}}$ on the planetary equator, $m_{i}$ is the ion mass, $e$ is the magnitude of the electron charge, and $c$ is the speed of light in vacuum. Therefore, the DA wave phase speed $V_{p}$ used in Saturnian Mach cone problems by Havnes et al. $[13,14]$ is not correct in general. The fluid ion response in Saturn's rings is also not valid for the DAWs whose wavelength is shorter than or comparable to $2 \pi \rho_{i} \simeq 290 \mathrm{~m}$. The wavelength of the DAWs participating in the formation of Mach cones is likely to be within a range of 20-50 m. Thus, Shukla and Mamun [18] employ 
a kinetic theory for the ion response, and present a generalized expression for the DAWs in magnetized dusty plasmas of Saturn's rings in order to clarify the role of short wavelength (in comparison with $\rho_{i}$ ) DAWs in the formation of Mach cones in dusty magnetoplasmas of Saturn's rings.

On the other hand, for typical dusty plasma parameters of Saturn's rings [17, 18, 19, 20, 21, 22, 23, 24], viz. $B_{0} \simeq 0.2 \mathrm{G}, T_{e}=15 \mathrm{eV}, T_{i}=10 \mathrm{eV}, n_{d 0} \simeq 10 \mathrm{~cm}^{-3}, n_{i 0} \simeq 10^{4} \mathrm{~cm}^{-3}$, $Z_{d} \simeq 10^{3}, r_{d} \simeq 0.25 \mu \mathrm{m}$, where $r_{d}$ is the dust particle radius, one finds that $C_{d}^{2} / V_{A d}^{2} \simeq 5 \times 10^{-5}$, where $V_{A d}=B_{0} / \sqrt{4 \pi n_{d 0} m_{d}}$ is the dust-Alfvén speed, and $C_{d} \simeq\left(Z_{d}^{2} n_{d 0} T_{i} / n_{i 0} m_{d}\right)^{1 / 2}$ is the DA speed. It turns out that in Saturn's dusty rings, low-frequency (in comparison with the dust gyro-frequency), long wavelength (in comparison with the ion gyro-radius) slow dispersive dust magneto-acoustic (DM) waves could also be relevant for the Mach cone formation [25, 26]. Mamun et al. [25] have predicted the possibility for the formation of Mach cones associated with the slow DM waves (DMWs) propagating in Saturn's dusty rings, and discussed their basic features.

Mach cones have also been observed in laboratory dusty plasmas $[4,7,9]$. The experimentally observed Mach cones [4, 7] in a dusty plasma have been theoretically interpreted by Dubin [6] in terms of constructive interference between dispersive dust lattice/acoustic-like waves. Because of the low dust-temperature, short inter-grain distance, and high dust grain charge, the inter-grain (screened) Coulomb coupling parameter $\Gamma \simeq Z_{d}^{2} e^{2} \exp \left(-a_{d} / \lambda_{D}\right) / a_{d} T_{d}$ can be much larger than one and the average inter-grain spacing could be of the order of the ion gyro-radius $\rho_{i}$, where $a_{d}=\left(3 / 4 \pi n_{d 0}\right)^{1 / 3}$ is the average inter-grain spacing, $\lambda_{D}$ is the characteristic Debye radius in dusty plasmas [24], and $T_{d}$ is the dust temperature. Recently, Mamun et al. [27] have presented a theory for the Mach cones in a strongly coupled dusty magnetoplasma which is composed of weakly coupled magnetized electrons and ions, and strongly coupled unmagnetized charged dust grains. They have derived the dispersion relations for the low-frequency (in comparison with the ion gyro-frequency) dusty plasma waves that are responsible for the formation of Mach cones in strongly coupled dusty magnetoplasmas. Since the formation of Mach cones in dusty magnetoplasmas has recently become an important issue from both theoretical $[13,14,15,18$, $25,26,27,28,29]$ and experimental $[4,7,9]$ points of view, in our present manuscript we systematically and rigorously study the possibility for the formation of Mach cons in space and laboratory dusty magnetoplasmas.

The manuscript is organized as follows. The possibility for the formation of Mach cones associated with different types of waves (viz. DAWs, DMWs, etc.) propagating in space dusty magnetoplasmas (particularly, in Saturn dusty rings) have been studied in Sec. 2. The possibility for formation of different types of Mach cones (viz. modified DA Mach cones, osillonic Mach cones, etc.) in laboratory dusty magneoplasmas are investigated in Sec. 3. Finally, a brief discussion is given in Sec. 4.

\section{Space dusty magnetoplasmas}

To study the possibility for the formation of Mach cones in space dusty magnetoplasmas, particularly in Saturn's dusty rings, we analyze first the dynamics of dust particles, and then different 
types of dust-associated waves that are responsible for the formation of Mach cones in Saturn's dusty rings.

\subsection{Dust dynamics}

We consider a charged dust particle of mass $m_{d}$ and charge $s Z_{d} e$ (where $s=-1$ for negatively charged dust and $s=+1$ for positively charged dust) moving in a field which includes Keplerian gravity, corotating planetary magnetic field with concomitant induced electric field $[17,18,19,20,21,22]$. We consider single particle dynamics and neglect the radiation pressure, plasma drag, planetary oblateness, charge fluctuations, and collective effects. The dynamics of such a negatively charged dust particle is governed by the combined gravitational, magnetic, and electric forces. The orbital angular velocity $\omega_{d}$ of this negatively charged dust particle can, therefore, be expressed as $[17,21]$

$$
\omega_{d}=\frac{1}{2 r^{3}}\left[s \omega_{c d} \pm \sqrt{\omega_{c d}^{2}+4 r^{3}\left(\Omega_{k}^{2}-s \omega_{c d} \Omega_{p}\right)}\right]
$$

where $r$ is the dust particle position normalized by the planet radius $R_{p}, \omega_{c d}=Z_{d} e B_{0} / m_{d} c$ and $\Omega_{k}=\left(G M_{p} / R_{p}^{3}\right)^{1 / 2}$ are the dust gyro and Kepler frequencies, both evaluated at a point on the planetary equator; $\Omega_{p}$ is the planetary spin rate, $M_{p}$ is the planet mass, and $G$ is the universal gravitational constant. We note that in deriving Eq. (1) the planetary magnetic field $\mathbf{B}_{p}$ is assumed to be dipolar with the dipole strength $\mathbf{M}_{s}=\mathbf{B}_{\mathbf{0}} R_{p}^{3}$, which is appropriate for Saturn and Jupiter. The $+(-)$ sign in Eq. (1) represents the prograde (retrograde) motion of the dust particle.

A large boulder and a small dust particle will, therefore, move at difference speeds. The difference in speeds $V_{d}$ is given by

$$
V_{d}=r R_{p}\left(\omega_{d}-r^{-3 / 2} \Omega_{k}\right) .
$$

To approximate $V_{d}$, let us consider a dust boulder in Saturn's rings $[13,17,19,21,22]: R_{p}=$ $60300 \mathrm{~km}, M_{p}=5.688 \times 10^{26} \mathrm{~kg}, \Omega_{p}=1.691 \times 10^{-4} \mathrm{rad} / \mathrm{s}, r \approx 2, B_{0} \simeq 0.2 \mathrm{G}, Z_{d} \simeq 750$, $r_{d} \simeq 0.5 \mu \mathrm{m}$, so that $\Omega_{k}=4.16 \times 10^{-4} \mathrm{~s}^{-1}$ and $\omega_{c d} \simeq 3.67 \times 10^{-5} \mathrm{~s}^{-1}$. So for a particle in

Saturn's rings we can safely take the approximations $\omega_{c d} \ll \Omega_{k}, \Omega_{p} \leq \Omega_{k}$, and $r \geq 1$, which allow us to approximate Eq. (2) as

$$
V_{d} \simeq \frac{s R_{p} \omega_{c d}}{2 r^{2}}\left(1-\frac{\Omega_{p}}{\Omega_{k}} r^{3 / 2}\right)
$$

Equation (3) represents an approximate expression for the speed of a dust boulder moving in Saturn's equatorial plane under the combined effects of the gravitational, magnetic, and electric forces.

\subsection{DA Mach cones}

We consider two kinds of dusty manetoplasmas that may exist in Saturn's dusty rings, viz. i) electron-ion-dust (EID) plasma (containing electrons, ions, and negatively charged dust [17, 18, 
19, 20, 21, 22]) and ii) electron-dust (ED) plasma (containing elctrons and positively charged dust $[30,31,32])$.

\section{i) EID plasmas}

We consider arbitrary wavelength DAWs which may participate in the formation of Mach cones in an EID plasma. Thus, at equilibrium we have $n_{i 0}=n_{e 0}+Z_{d} n_{d 0}$. When the wavelength of the DAWs is comparable or shorter than the ion gyroradius, we use the ion Vlasov equation to calculate the ion density perturbation. So for $\omega \ll \omega_{c i}$ we have $n_{i 1}=-\left(k^{2} / 4 \pi e\right) \chi_{i} \phi$, where the ion susceptibility involving two-dimensional ion motions is [33, 34]

$$
\chi_{i} \simeq \frac{1}{k^{2} \lambda_{D i}^{2}}\left[1-\Gamma_{0}\left(b_{i}\right)+2 \Gamma_{1}\left(b_{i}\right) \frac{\omega^{2}}{\omega_{c i}^{2}}\right],
$$

where $\lambda_{D i}=\left(T_{i} / 4 \pi n_{i 0} e^{2}\right)^{1 / 2}, \Gamma_{0,1}=I_{0,1} \exp \left(-b_{i}\right), b_{i}=k_{\perp}^{2} \rho_{i}^{2}$, and $I_{0}\left(I_{1}\right)$ is the zero (first) order modified Bessel function.

On the other hand, for $\omega \ll k_{z} v_{t e}, \omega_{c e} k_{z} / k_{\perp}$ and $b_{e}=k_{\perp}^{2} v_{t e}^{2} / \omega_{c e}^{2} \ll 1$, the electrons rapidly thermalize along $\hat{\mathbf{z}}$ and establish a Boltzmann distribution. The corresponding electron density perturbation is $n_{e 1}=\left(k^{2} / 4 \pi e\right) \chi_{e} \phi$, where the electron susceptibility is

$$
\chi_{e} \simeq \frac{1}{k^{2} \lambda_{D e}^{2}}
$$

where $v_{t e}=\left(T_{e} / m_{e}\right)^{1 / 2}, \omega_{c e}=e B_{0} / m_{e} c, \lambda_{D e}=\left(T_{e} / 4 \pi n_{e 0} e^{2}\right)^{1 / 2}$, and $m_{e}$ is the electron mass. For $\omega_{c d} \ll \omega$ the dust grains can be treated as unmagnetized. The corresponding dust number density perturbation is $n_{d 1}=\left(k^{2} / 4 \pi Z_{d} e\right) \chi_{d} \phi$, where the dust susceptibility is

$$
\chi_{d} \simeq-\frac{\omega_{p d}^{2}}{\omega^{2}-3 k^{2} V_{T d}^{2}},
$$

where $\omega_{p d}=\left(4 \pi n_{d 0} Z_{d}^{2} e^{2} / m_{d}\right)^{1 / 2}$ is the dust plasma frequency and $V_{T d}=\left(T_{d} / m_{d}\right)^{1 / 2}$ is the dust thermal speed. Now, using Eqs. (4)-(6) the dispersion relation $1+\chi_{e}+\chi_{i}+\chi_{d}=0$ can be expressed in the form [18]

$$
\left[1-\frac{1}{\alpha} \Gamma_{0}\left(b_{i}\right)\right] \omega^{2}-\frac{2}{\alpha} \Gamma_{1}\left(b_{i}\right) \frac{\omega^{4}}{\omega_{c i}^{2}}=k^{2} C_{D}^{2},
$$

where $\alpha=1+k^{2} \lambda_{D i}^{2}+n_{e 0} T_{i} / n_{i 0} T_{e}$. Equation (7) is the general dispersion relation for the DAWs in a magnetized EID plasma including the effect of arbitrary $b_{i}$. This is valid for arbitrary (short or long) wavelength DAWs. In a short wavelength limit $\left(b_{i} \gg 1\right)$, Eq. (7) reduces to $\omega / k=C_{D}$, which coincides with the dispersion relation for DAWs of Rao et al. [16]. We note that in deriving Eq. (7) we assumed that $\omega / k \gg V_{T d}$.

We are now in a position to analyze the possibility for the formation of Mach cones by DAWs [defined by Eq. (7)]: The Mach cones can be formed if $V_{d}$ [defined by Eq. (3) with $s=-1$ ] is larger than $V_{p}=\omega / k$ [defined by Eq. (7)], i.e. $V_{d} / V_{p}>1$. If this condition is satisfied, the Mach cone opening angle is given by $\theta=\sin ^{-1}\left(V_{p} / V_{d}\right)$. We analyze $V_{d}$ and $V_{p}$ by solving numerically Eqs. (3) and (7). The numerical results are displayed in figures 1 and 2. The upper plot of figure 

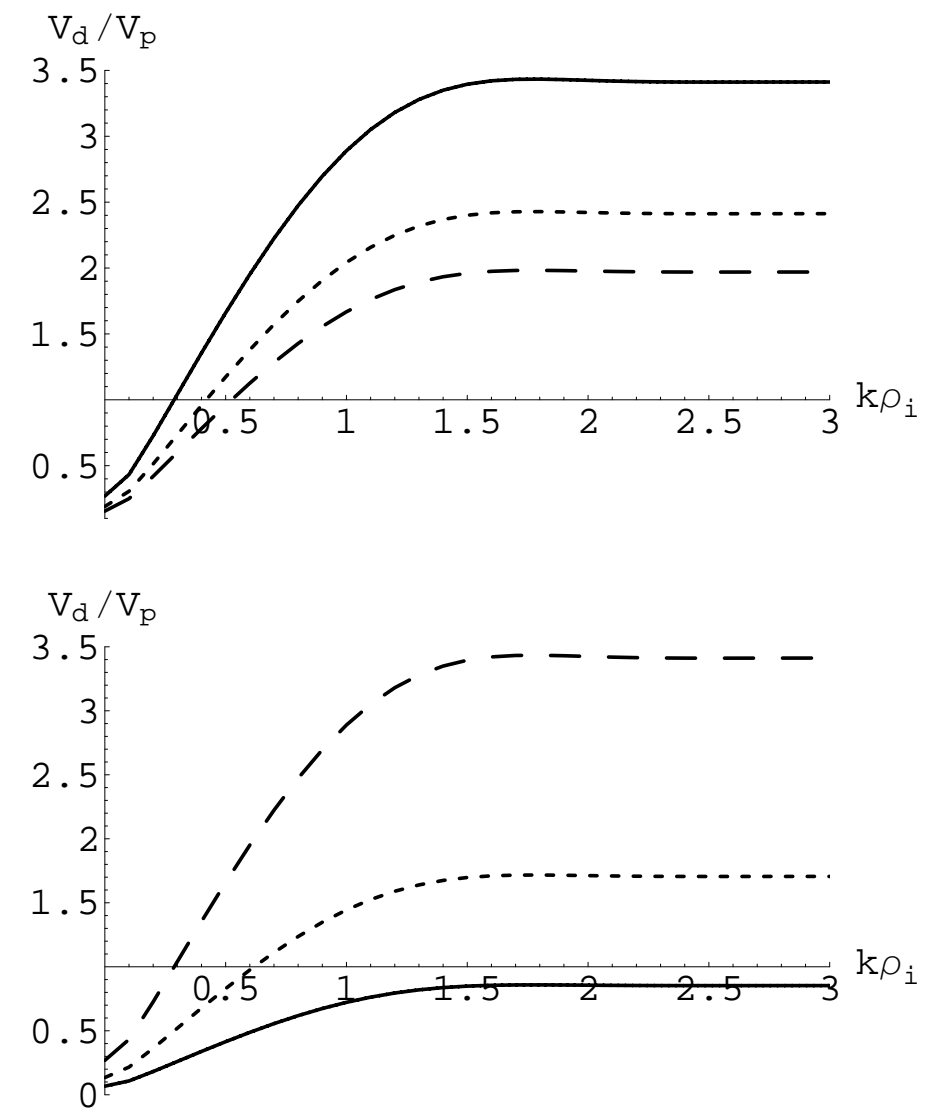

Figure 1: Showing $V_{d} / V_{p}$ vs. $k \rho_{i}$ curves for $n_{d 0}=10 \mathrm{~cm}^{-3}, Z_{d}=10^{3}, T_{e}=10 T_{i}, n_{e 0}=10^{3} \mathrm{~cm}^{-3}, r=2$, $r_{d}=0.25 \mu \mathrm{m}$, and $\delta=85^{\circ}$ and for different values of $B_{0}$ and $T_{i}$. The upper plot, where $B_{0}=0.1 \mathrm{G}, T_{i}=5 \mathrm{eV}$ (solid curve), $T_{i}=10 \mathrm{eV}$ (dotted curve), and $T_{i}=15$ (dashed curve), shows the effect of $T_{i}$ on $V_{d} / V_{p}$ vs. $k \rho_{i}$ curve. The lower plot, where $T_{i}=10 \mathrm{eV}, B_{0}=0.05 \mathrm{G}$ (solid curve), $B_{0}=0.1 \mathrm{G}$ (dotted curve), and $B_{0}=0.15$ $\mathrm{G}$ (dashed curve), shows the effect of $B_{0}$ on $V_{d} / V_{p}$ vs. $k \rho_{i}$ curve.

1 shows that as we increase the ion temperature, $V_{d} / V_{p}$ decreases. This implies that higher ion temperature is not in favor of the formation of the DA Mach cones. The lower plot of figure 1 shows that the condition for the Mach cone formation $\left(V_{d} / V_{p}>1\right)$ is not satisfied for $B_{0}=0.05$ G. However, if $B_{0}$ is further increased, DA Mach cones are found to be formed. This means that the increase in the ambient magnetic field, which increases $V_{d}$ (found to be $10.3 \mathrm{~m} / \mathrm{s}, 20.6 \mathrm{~m} / \mathrm{s}$, and $41.2 \mathrm{~m} / \mathrm{s}$ for the planetary magnetic field of $0.05 \mathrm{G}, 0.1 \mathrm{G}$, and $0.2 \mathrm{G}$, respectively) is in the favor of the formation of the modified DA Mach cones. The upper plot of figure 2 shows that as we increase the magnitude of the dust charge, $V_{d} / V_{p}$ increases. This means that more negatively charged dust particles are in favor of the formation of the short wavelength DA Mach cones. The lower plot of figure 2 shows that as we increase the dust grain radius, $V_{d} / V_{p}$ decreases. This means that larger dust particles are not in favor of the formation of the DA Mach cones [13]. From figures 1 and 2 it is also obvious how the Mach cone opening angle $\theta=\sin ^{-1}\left(V_{p} / V_{d}\right)$ varies with $k, B_{0}, T_{i}, r_{d}$, and $Z_{d}$. For Saturn's plasma parameters, we have estimated the Mach cone opening angle $\theta$ associated with the modified DAWs of wavelength $\sim 30 \mathrm{~m}$ propagating at an angle $\delta=85^{\circ}$ against the magnetic field direction. These are $\sim 38^{\circ}$ and $\sim 17^{\circ}$ for $B_{0}=0.1 \mathrm{G}$ 

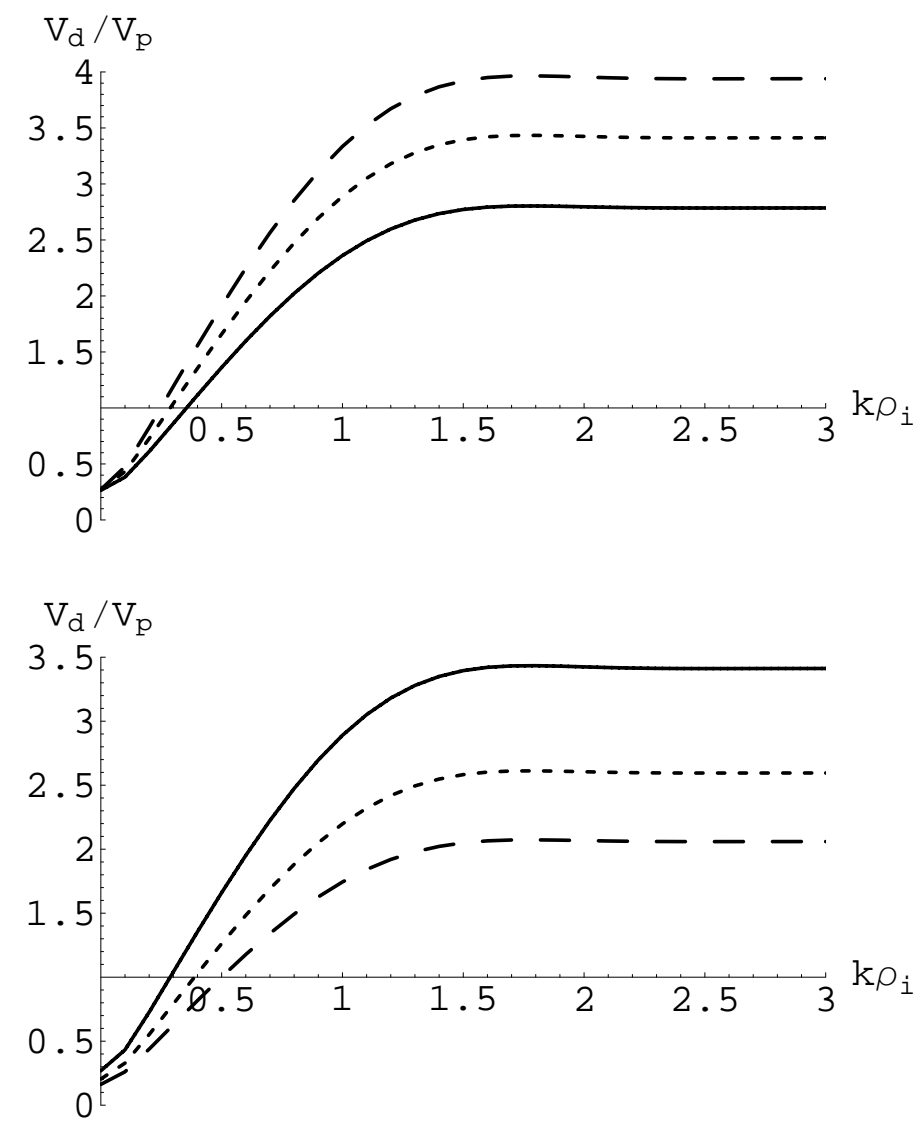

Figure 2: Showing $V_{d} / V_{p}$ vs. $k \rho_{i}$ curves for $n_{d 0}=10 \mathrm{~cm}^{-3}, B_{0}=0.1 \mathrm{G}, T_{i}=10 \mathrm{eV}, T_{e}=10 T_{i}, n_{e 0}=10^{3} \mathrm{~cm}^{-3}$, $r=2$, and $\delta=85^{\circ}$ and for different values of $r_{d}$ and $Z_{d}$. The upper plot, where $r_{d}=0.25 \mu \mathrm{m}, Z_{d}=500$ (solid curve), $Z_{d}=1000$ (dotted curve) and $Z_{d}=5000$ (dashed curve), shows the effects of $Z_{d}$ on $V_{d} / V_{p}$ vs. $k \rho_{i}$ curve. The lower plot, where $Z_{d}=10^{3}, r_{d}=0.25 \mu \mathrm{m}$ (solid curve), $r_{d}=0.35 \mu \mathrm{m}$ (dotted curve), and $r_{d}=0.5 \mu \mathrm{m}$ (dashed curve), shows the effect of $r_{d}$ on $V_{d} / V_{p}$ vs. $k \rho_{i}$ curve.

and $B_{0}=0.2 \mathrm{G}$, respectively.

\section{ii) ED plasmas}

We now consider the propagation of low-frequency $\left(\omega \ll \omega_{c e}\right)$ electrostatic waves in an ED plasma $[30,31,32,35,36,37]$. Thus, at equilibrium we have $n_{e 0} \simeq Z_{d} n_{d 0}$.

For waves of arbitrary wavelength and arbitrary electron thermal gyro-radius $\rho_{e}=v t e / \omega_{c e}$, we employ a kinetic theory [33], and express the electron density perturbation as $n_{e 1}=\left(k^{2} / 4 \pi e\right) \chi_{e} \phi$, where the electron susceptibility (for two-dimensional electron motion in a plane perpendicular to $\hat{\mathbf{z}}$ and for $\left.\omega \ll \omega_{c e}\right)$ is

$$
\chi_{e} \simeq \frac{1}{k^{2} \lambda_{D e}^{2}}\left[1-\Gamma_{0}\left(b_{e}\right)+2 \Gamma_{1}\left(b_{e}\right) \frac{\omega^{2}}{\omega_{c e}^{2}}\right]
$$

where $\Gamma_{0,1}=I_{0,1} \exp \left(-b_{e}\right), I_{0}\left(I_{1}\right)$ is the modified Bessel function of zero (first) order, and $b_{e}=k^{2} \rho_{e}^{2}$.

For $\omega_{c d} \ll \omega$ the dust grains can be treated as unmagnetized, and the dust susceptibility $\chi_{d}$ 
is given by Eq. (6). Thus, using Eqs. (6) and (8) in $1+\chi_{e}+\chi_{d}=0$, the dispersion relation involving $V_{p}=\omega / k$ can be expressed in the form [28]

$$
\left[\alpha-\Gamma_{0}\left(b_{e}\right)\right] V_{p}^{2}+2 \Gamma_{1}\left(b_{e}\right) \frac{k^{2} V_{p}^{4}}{\omega_{c e}^{2}}=C_{D}^{2},
$$

where $C_{D}=\lambda_{D e} \omega_{p d}=\left(Z_{d} T_{e} / m_{d}\right)^{1 / 2}$ is the DA speed [35] and $\alpha=1+k^{2} \lambda_{D e}^{2}$. We note that in deriving Eq. (9), we assumed that $\omega / k \gg V_{T d}$. Equation (9) is the general dispersion relation for the modified DAWs [35] in a magnetized ED plasma including finite electron gyro-radius effect. This is valid for arbitrary (short or long) wavelength modified DAWs.

The Mach cones can be formed by the modified DAWs defined by Eq. (9) if $V_{d}$ [defined by Eq. (3) with $s=+1$ ] is larger than the modified DAW phase speed $V_{p}$ [defined by Eq. (9)], i.e. $V_{d} / V_{p}>1$. If this condition is satisfied, the Mach cone opening angle is $\theta=\sin ^{-1}\left(V_{p} / V_{d}\right)$, where $V_{d}$ is given by Eq. (3) with $s=+1$, and $V_{p}=\omega / k$ is defined by Eq. (9). It is obvious that Mach cones can only be formed by those dust particles which are either inside $\left(r<r_{0}\right)$ or outside $\left(r>r_{0}\right)$ the synchronous distance $r_{0}$, a value of $r$ for which $V_{d}=0$. Thus, from Eq. (3) with $s=+1$, the synchronous distance $r_{0}$ can be estimated as $r=r_{0}=\left(\Omega_{k} / \Omega_{p}\right)^{2 / 3}$. It is found that $r_{0}=1.822$ for Saturn.

To analyze the possibility for the formation of Mach cones associated with the modified DAWs defined by (9), we analyze $V_{d}$ and $V_{p}$ by solving numerically Eqs. (3) and (9) for typical ED plasma parameters of Saturn [17, 21, 24, 23]: $T_{e}=100 \mathrm{eV}, n_{d 0}=10 \mathrm{~cm}^{-3}, Z_{d}=100$, $B_{0}=0.2 \mathrm{G}, M_{p}=5.688 \times 10^{26} \mathrm{~kg}, R_{p}=60300 \mathrm{~km}$, and for dust particles inside $\left(r<r_{0}\right)$ the synchronous distance $r_{0}$. The numerical results are displayed in figure 3 . The upper plot of figure 3 shows that for $r_{d}=1 \mu \mathrm{m}$, the maximum wavelength of the modified DAWs, for which the Mach cones are formed, are $\sim 6.81 \mathrm{~m}, \sim 3.94 \mathrm{~m}$, and $\sim 1.92 \mathrm{~m}$ for $r=1.5, r=1.6$, and $r=1.7$, respectively. The lower plot of figure 1 indicates that for $r=1.6$, the maximum wavelength are $\sim 10.7 \mathrm{~m}, \sim 3.94 \mathrm{~m}$, and $\sim 2.08 \mathrm{~m}$ for $r_{d}=0.5 \mu \mathrm{m}, r_{d}=1 \mu \mathrm{m}$, and $r_{d}=1.5 \mu \mathrm{m}$, respectively.

We have also analyzed the Mach cone formation at locations outside $\left(r>r_{0}\right)$ the synchronous distance $r_{0}$. Our analysis reveals that for $r_{d}=1 \mu \mathrm{m}$, the maximum wavelength of the modified DAWs, for which the Mach cones are formed, are $\sim 1 \mathrm{~m}, \sim 2.14 \mathrm{~m}$, and $\sim 3 \mathrm{~m}$ for $r=1.9, r=2.0$, and $r=2.1$, respectively. We have also found that for $r=2$, the maximum wavelength are $\sim 6$ $\mathrm{m}, \sim 2.14 \mathrm{~m}$, and $\sim 1.15 \mathrm{~m}$ for $r_{d}=0.5 \mu \mathrm{m}, r_{d}=1 \mu \mathrm{m}$, and $r_{d}=1.5 \mu \mathrm{m}$, respectively.

Figure 3 also shows how the Mach cone opening angle $\theta=\sin ^{-1}\left(V_{p} / V_{d}\right)$ varies with $k \rho_{e}, r$, and $r_{d}$. We have estimated the Mach cone opening angle $\theta$ associated with the modified DAWs of wavelength $\sim 7.5 \mathrm{~m}$ (corresponding to $k \rho_{e} \simeq 1$, since $\rho_{e} \simeq 1.193 \mathrm{~m}$ for $T_{e}=100 \mathrm{eV}$ and $B_{0}=0.2$ G) in both cases of $r=1.5<r_{0}$ and $r=2.5>r_{0}$ for $r_{d}=0.5 \mu \mathrm{m}$. This is found to be $\sim 25^{\circ}$ and $\sim 29^{\circ}$ in the cases of $r=1.5<r_{0}$ and $r=2.5>r_{0}$, respectively.

\subsection{DM Mach Cones}

We are now interested in examining the possibility for the formation of Mach cones involving obliquely propagating low-frequency $\left(\omega \ll \omega_{c d}\right)$, long wavelength $\left(k_{\perp}^{2} \rho_{i}^{2} \ll 1\right)$ DMWs in dusty magnetoplasmas of Saturn's rings. To avoid mathematical complexities, we consider two- 

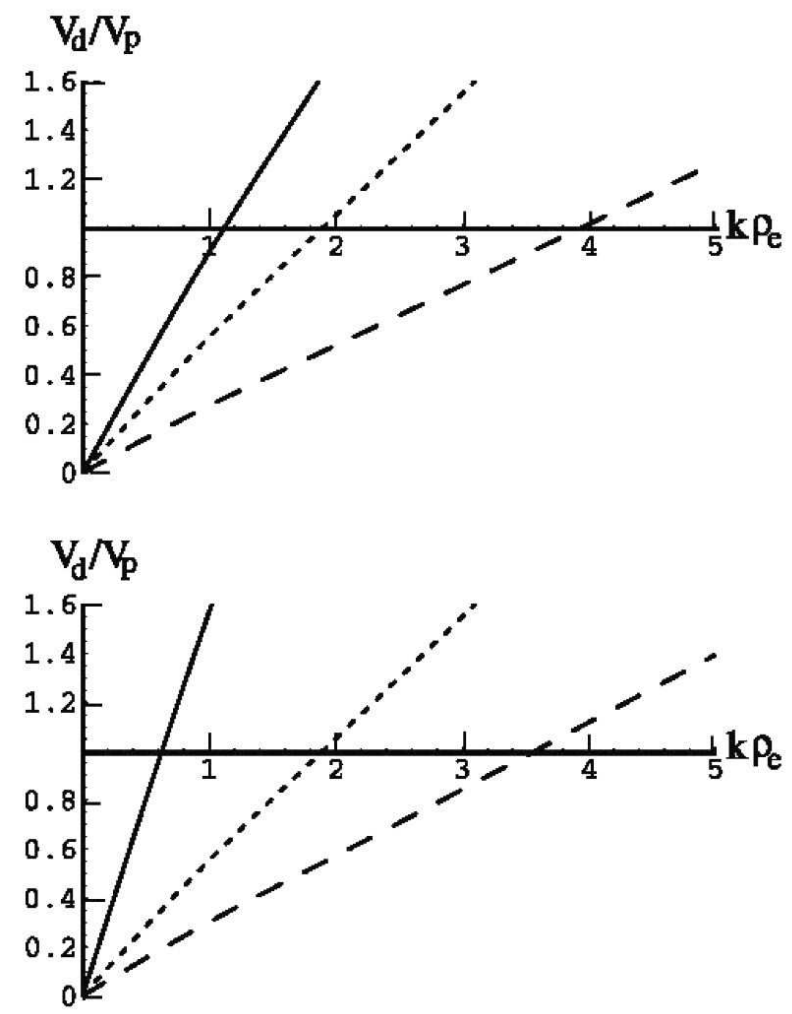

Figure 3: Showing $V_{d} / V_{p}$ versus $k \rho_{e}$ curves for $r<r_{0}=1.822$ with parameters (given in the text), and for different values of $r$ and $r_{d}$. The upper plot, where $r_{d}=1 \mu \mathrm{m}, r=1.5$ (solid curve), $r=1.6$ (dotted curve), and $r=1.7$ (dashed curve), shows the effect of $r$ on $V_{d} / V_{p}$ versus $k \rho_{e}$ curve. The lower plot, where $r=1.6$, $r_{d}=0.5 \mu \mathrm{m}$ (solid curve), $r_{d}=1 \mu \mathrm{m}$ (dotted curve), and $r_{d}=1.5 \mu \mathrm{m}$ (dashed curve), shows the effect of $r_{d}$ on $V_{d} / V_{p}$ versus $k \rho_{e}$ curve.

component magnetized dusty plasma composed of negatively charged dust grains and positively charged ions. We consider a small amplitude perturbation in such a magnetized dust-ion plasma, which may be described by two linearized coupled equations [25]

$$
\begin{gathered}
\frac{\partial^{2} \mathbf{u}_{d}}{\partial t^{2}}+\frac{V_{A d}^{2}}{\omega_{c i}} \nabla \times \frac{\partial^{2} \mathbf{B}}{\partial t^{2}}-V_{A d}^{2}\left(\nabla \times \frac{\partial \mathbf{B}}{\partial t}\right) \times \hat{\mathbf{z}} \\
\left.-C_{d}^{2} \nabla\left(\nabla \cdot \mathbf{u}_{d}\right)\right]=0, \\
\frac{\partial \mathbf{B}}{\partial t}+\hat{\mathbf{z}}\left(\nabla \cdot \mathbf{u}_{d}\right)-(\hat{\mathbf{z}} \cdot \nabla) \mathbf{u}_{d}-\frac{1}{\omega_{c d}} \nabla \times \frac{\partial \mathbf{u}_{d}}{\partial t}=0 .
\end{gathered}
$$

Now, using Eqs. (10) and (11) we obtain three types of obliquely propagating DMWs. These are [25]

$$
\frac{\omega}{k}=\frac{V_{A d} \cos \delta}{\sqrt{1+k^{2} \lambda_{i}^{2}}}
$$

and

$$
\begin{aligned}
& \frac{\omega}{k}=\frac{1}{\sqrt{2}}\left[\frac{V_{A d}^{2}}{1+k^{2} \lambda_{i}^{2}}+C_{d}^{2}\right. \\
& \left. \pm \sqrt{\left(\frac{V_{A d}^{2}}{1+k^{2} \lambda_{i}^{2}}+C_{d}^{2}\right)^{2}-\frac{4 V_{A d}^{2} C_{d}^{2} \cos ^{2} \delta}{1+k^{2} \lambda_{i}^{2}}}\right]^{1 / 2}
\end{aligned}
$$


where $\lambda_{i}=c / \omega_{p i}$ is the ion skin depth and $\delta$ is the angle between the directions of the ambient magnetic field and the wave propagation.

Equation (12) represents the dispersion relation for the shear dust-Alfvén waves modified by the effect of the ion-skin depth which decreases their phase speed by the factor $\sqrt{1+k^{2} \lambda_{i}^{2}}$. On the other hand, Eq. (13) with $+(-)$ sign represents the dispersion relation for the fast (slow) DMW modified by the effect of the ion-skin depth and obliqueness. For typical plasma parameters of Saturn's dusty rings $[17,19,20,21,22,24,23]$, we find that $C_{d}^{2} / V_{A d}^{2} \simeq 3.7 \times 10^{-5}$. It is then likely that Mach cones in Saturn's dusty rings may also involve long wavelength DMWs.

We numerically analyze $V_{d} / V_{p}$ for typical plasma parameters corresponding to Saturn's dusty rings. The numerical results are depicted in figures 4 and 5.

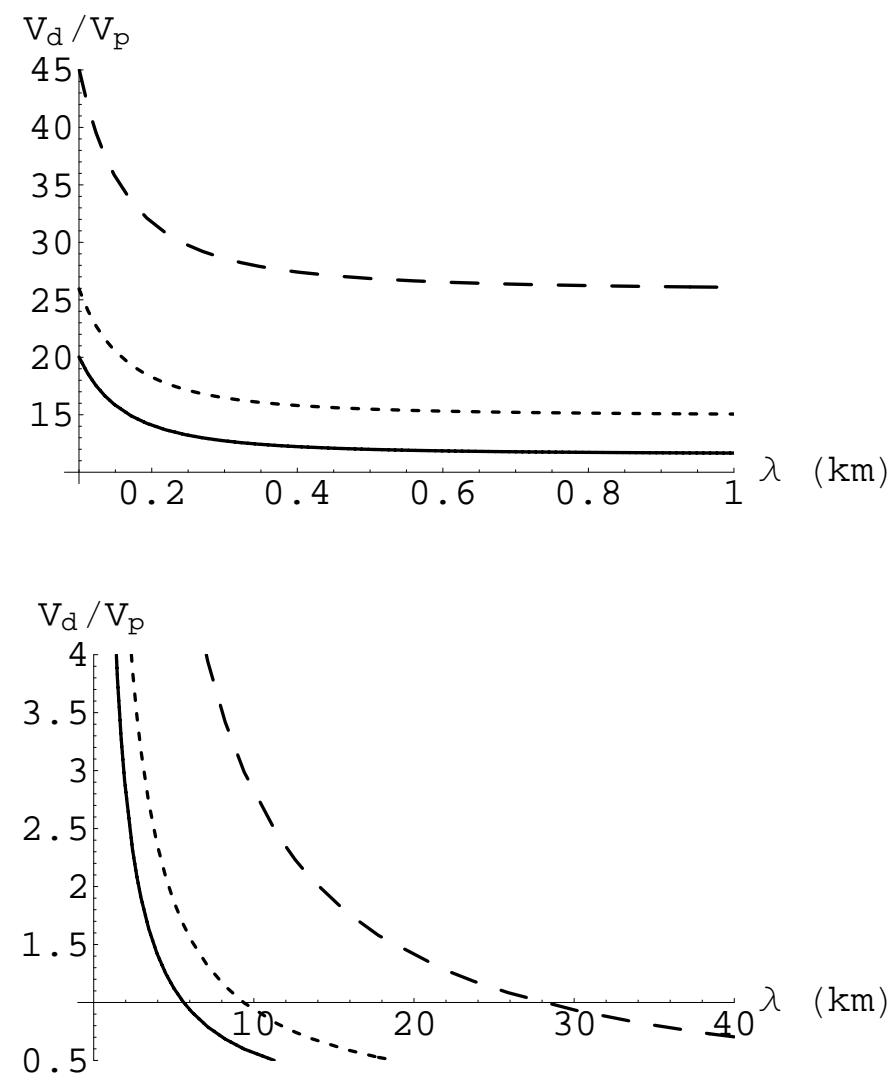

Figure 4: Showing the wavelength regimes of slow DMWs (upper plot) and of shear dust-Alfvén waves (lower plot) propagating with different angle $\delta$ for which Mach cones are formed in Saturn's dusty rings $\left(B_{0}=0.2\right.$ G, $\left.T_{i}=10 \mathrm{eV}, n_{d}=10 \mathrm{~cm}^{-3}, Z_{d}=750, r=2, r_{d}=0.5 \mu \mathrm{m}\right)$. The solid, dotted and dashed curves are for $\delta=85^{\circ}$, $\delta=87^{\circ}$, and $\delta=89^{\circ}$ in both the plots.

Figure 4 shows that Mach cones are formed by the shear dust-Alfvén waves of wavelength $\sim 7$ $\mathrm{km}$ or less (viz. lower plot), and by slow DMWs of without the upper bound on the wavelength (viz. upper plot). We also find that the upper bound on the wavelength of both the shear dust-Alfvén waves and slow DMWs by which Mach cones are formed increases as we increase their propagation angle $\delta$ (viz. lower and upper plots of figure 4). We observe here that for the 

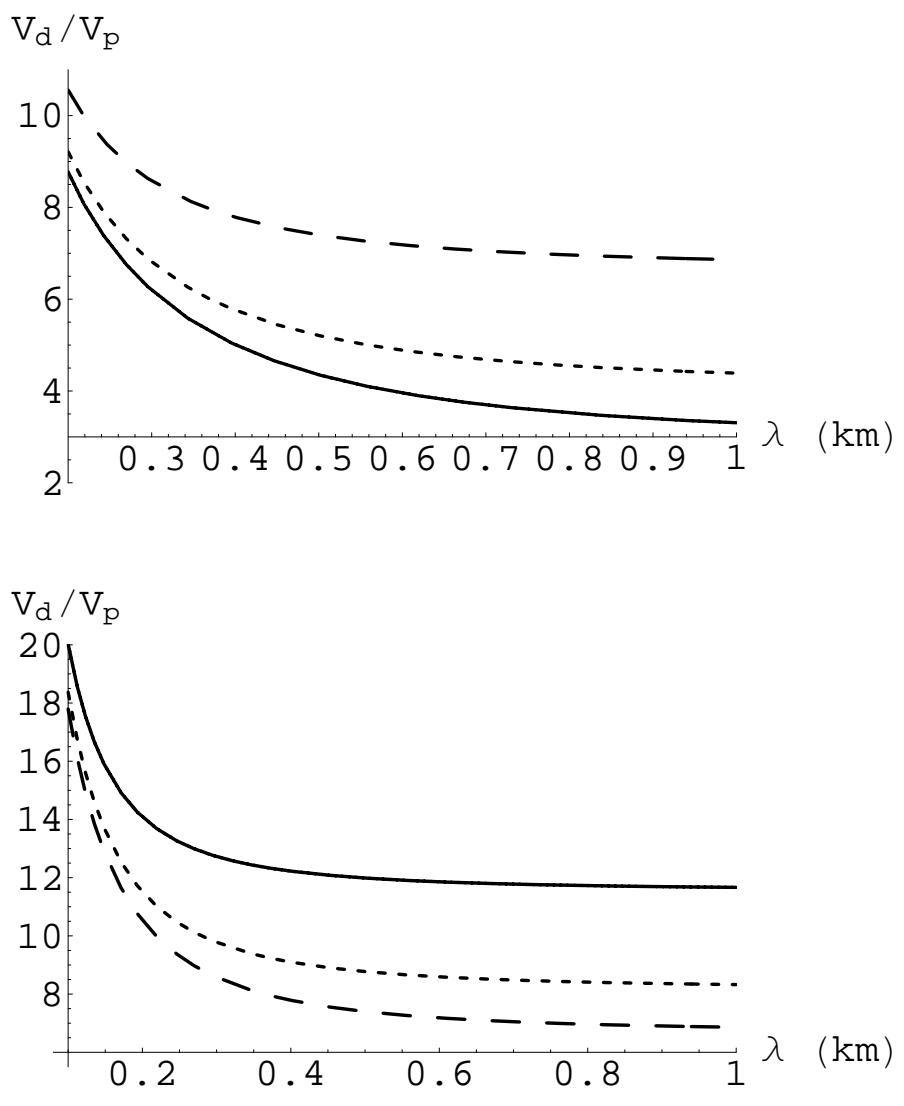

Figure 5: Showing the wavelength regimes of slow DMWs for which Mach cones are formed in Saturn's dusty rings $\left(n_{d 0}=10 \mathrm{~cm}^{-3}, Z_{d}=750, r=2, r_{d}=0.5 \mu \mathrm{m}\right.$, and $\left.\delta=85^{\circ}\right)$. The upper plot, where $T_{i}=10 \mathrm{eV}, B_{0}=0.05$ $\mathrm{G}$ (solid curve), $B_{0}=0.1 \mathrm{G}$ (dotted curve) and $B_{0}=0.2 \mathrm{G}$ (dashed curve), shows the effect of $B_{0}$ on $V_{d} / V_{p}$ vs. $\lambda$ curve. The lower plot, where $B_{0}=0.2 \mathrm{G}, T_{i}=10 \mathrm{eV}$ (solid curve), $T_{i}=20 \mathrm{eV}$ (dotted curve), and $T_{i}=30 \mathrm{eV}$ (dashed curve), shows the effect of $T_{i}$ on $V_{d} / V_{p}$ vs. $\lambda$ curve.

plasma parameters of Saturn's dusty rings, Mach cones are not formed by the fast DMWs.

We also examine the effects of the ion temperature $T_{i}$ and the ambient magnetic field $B_{0}$ on the formation of Mach cones associated with the shear dust-Alfvén waves and slow DMWs. We find that the ion temperature $T_{i}$ and the ambient magnetic field $\left(B_{0}\right)$ have an insignificant effect on the formation of Mach cones and their opening angles associated with the shear dust-Alfvén waves. However, these do have significant effects on those associated slow DMWs. These are depicted in figure 5 . It is seen that as we increase (decrease) $B_{0}\left(T_{i}\right)$ the maximum wavelength for which Mach cones are formed increases, but the opening angle decreases.

Figures 4 and 5 also imply how the Mach cone opening angle $\theta=\sin ^{-1}\left(V_{p} / V_{d}\right)$ varies with $\lambda, \delta, B_{0}$, and $T_{i}$. For Saturn's ring parameters, we have estimated the Mach cone opening angle $\theta$ associated with the shear dust-Alfvén waves and slow DMWs of wavelength of $5 \mathrm{~km}$, propagating with an angle $\delta=85^{\circ}$. These are $\sim 32^{\circ}$ and $\sim 4^{\circ}$, respectively.

\section{Laboratory dusty magnetoplasmas}

We now focus on the possibility for the formation of Mach cones in laboratory dusty magne- 
toplasmas. We first consider a strongly coupled dusty magnetoplasma, and present the theory for the formation of Mach cones associated with modified DA waves. We then consider a dusty magnatoplasma with elongated (rod-like) dust grains, and analyze the possibility for the formation of oscillonic Mach cones.

\subsection{Modified DA Mach cones}

We consider the propagation of low-frequency $\left(\omega_{c d} \ll \omega \ll \omega_{c i}\right)$ electrostatic perturbations in a strongly coupled dusty magnetoplasma containing weakly coupled magneized electrons and ions, and strongly coupled unmagnetized dust. For waves of arbitrary wavelength and ion gyroradius $\rho_{i}$, we use a kinetic theory in order to calculate the ion density perturbation. Thus, as before, for $\omega \ll \omega_{c i}$ the ion susceptibility $\chi_{i}$ involving two-dimensional (in a plane perpendicular to the external magnetic field direction) weakly coupled ion motions is given by Eq. (4).

On the other hand, for $b_{e}=k^{2} v_{t e}^{2} / \omega_{c e}^{2} \ll 1$, the electron density perturbation is given by $n_{e 1}=\left(k^{2} / 4 \pi e\right) \chi_{e} \phi$, where the electron susceptibility, for $k_{z} v_{t i} \ll \omega$ and $k_{z} / k \ll \omega / \omega_{c e} \ll 1$, is

$$
\chi_{e} \simeq \frac{\omega_{p e}^{2}}{\omega_{c e}^{2}} .
$$

The quasi-localized charge approximation (QLCA) [38], and the generalized hydrodynamic model (GHM) [39] allow us to express the dust number density perturbation as

$$
n_{d 1}=\left(k^{2} / 4 \pi Z_{d} e\right) \chi_{d} \phi
$$

where the dust susceptibility in a collisionless limit is $[38,39,40]$

$$
\chi_{d} \simeq-\frac{\omega_{p d}^{2}}{\omega^{2}-\omega_{p d}^{2} \mathcal{D}(k)} .
$$

We note that in Eq. (15) the $\mathcal{D}(k)$-term arises due to the strong correlation between dust particles. We now approximate $\mathcal{D}(k)$ by the QLCA in the limit $\left(\Gamma_{d} \gg 1\right.$ and $\left.d \leq \lambda_{d}\right)$ considered by Rosenberg and Kalman [38], and the GHM in the limit $(1 \leq \Gamma \leq 200)$ considered by Kaw and Sen [39]. That is, using the QLCA and long wavelength (in comparison with $\lambda_{D}$ ) limit, we can approximate $\mathcal{D}(k)$ as

$$
\mathcal{D}(k) \equiv \mathcal{D}_{R K}(k) \simeq f k^{2} a_{d}^{2}
$$

where $f=-(4 / 45)\left(0.9+0.05 a_{d}^{2} / \lambda_{D}^{2}\right)$ for $\Gamma_{d} \gg 1$ and $d \leq \lambda_{D}$. On the other hand, using the GHM we can approximate $\mathcal{D}(k)$ as

$$
\mathcal{D}(k) \equiv \mathcal{D}_{K S}(k) \simeq \gamma_{d} \mu_{d} k^{2} \lambda_{D d}^{2},
$$

where $\gamma_{d}$ is the adiabatic index, $\lambda_{D d}=\left(T_{d} / 4 \pi n_{d 0} Z_{d}^{2} e^{2}\right)^{1 / 2}$, and $\mu_{d}=1+(1 / 3) u(\Gamma)+(\Gamma / 9)[\partial u(\Gamma) / \partial \Gamma]$ is the compressibility [41], and $u(\Gamma)$ is the excess thermal energy of the system, which is often obtained by fitting data from Monte Carlo and molecular dynamic simulations or experiments, and can be approximated $[42,40]$ as $u(\Gamma) \simeq-0.89 \Gamma+0.95 \Gamma^{1 / 4}+0.19 \Gamma^{-1 / 4}-0.81$ for $1<\Gamma<200$. 
Now, using Eqs. (4), (14), and (15) in $1+\chi_{e}+\chi_{i}+\chi_{d}=0$, the dispersion relation involving $\Psi=\omega / k V_{d}$ (a ratio of the wave phase speed $V_{p}=\omega / k$ to the dust particle speed $V_{d}$ ) can be expressed as $[28]$

$$
\alpha+\frac{2 V_{d}^{2} \Lambda_{1}}{\lambda_{D i}^{2} \omega_{c i}^{2}} \Psi^{2}-\frac{\omega_{p d}^{2}}{k^{2} V_{d}^{2} \Psi-\omega_{p d}^{2} \mathcal{D}(k)}=0,
$$

where $\alpha=1+\omega_{p e}^{2} / \omega_{c e}^{2}+\left(1-\Lambda_{0}\right) / k^{2} \lambda_{D i}^{2}$. We note that for the QLCA we replace $\mathcal{D}(k)$ by $\mathcal{D}_{R K}(k)$ defined by Eq. (16) and for the GHM we replace $\mathcal{D}(k)$ by $\mathcal{D}_{K S}(k)$ defined by Eq. (17). The dust particle speed $V_{d}$ in Eq. (18) is considered as a free parameter.

The Mach cones can be formed by the modified DAWs defined by Eq. (18) if $\Psi=V_{p} / V_{d}<1$. If this condition is satisfied, the Mach cone opening angle $\theta$ is given by $\theta=\sin ^{-1} \Psi$, where $\Psi$ is defined by Eq. (18). To analyze the possibility for the formation of the Mach cones associated with the waves defined by Eq. (18), we numerically analyze Eq. (18), and find $\Psi=1$ curves in $\left(V_{d}, k \rho_{i}\right)$ space for typical laboratory dusty plasma parameters [43]: $T_{e} \simeq 2 \mathrm{eV}, T_{i} \simeq 0.5 T_{e}$, $T_{d} \simeq 0.07 \mathrm{eV}, n_{i 0} \simeq 3 \times 10^{9} \mathrm{~cm}^{-3}, Z_{d} \simeq 10^{3}, n_{d 0} \simeq 2 \times 10^{5} \mathrm{~cm}^{-3}, r_{d} \simeq 0.3 \mu \mathrm{m}, B_{0}=10^{3} \mathrm{G}$, $B_{0}=5 \times 10^{3} \mathrm{G}$, and $B_{0}=10^{4} \mathrm{G}$. The numerical results are displayed in figure 6 . The $\Psi=1$ curves in $\left(V_{d}, k \rho_{i}\right)$ space will, obviously, determine the critical values of the dust particle speed $V_{d}$ and the corresponding wavelength $\lambda=2 \pi / k$ for which the Mach cones are formed. The regions above the $\Psi=1$ curves correspond to $\Psi<1$, i.e. correspond to a regime for which the Mach cones are formed in strongly coupled dusty magnetoplasmas. The upper plot of figure 6 shows the critical values of the dust particle speed $V_{d}$ and the corresponding wavelength $\lambda=2 \pi / k$ for which the Mach cones are formed for two different approaches, namely the QLCA and the GHM, and clearly indicates that for laboratory dusty magnetoplasma conditions [43] the QLCA and the GHM do not have any significant discrepancies, and for $k \rho_{i} \leq 1$ both approaches give exactly the same results.

The lower plot of figure 6 , where $\mathcal{D}=\mathcal{D}_{R K}$ is used, shows the critical values of the dust particle speed $V_{d}$ and the corresponding wavelength $\lambda=2 \pi / k$ for which the Mach cones are formed for different values of the external magnetic field strength. It implies that as we increase the magnitude of the external magnetic field, for the wave of fixed wavelength we need a dust particle of higher speed in order for the formation of the Mach cones.

\subsection{Oscillonic Mach cones}

We finally consider a dusty magnetoplasma containing electrons, ions and elongated (rodlike) negatively charged dust grains $[44,45,46,47,48]$, and study the possibility for the formation of Mach cones associated with oscillons, which are low-frequency $\left(\omega \ll \omega_{c i}\right)$ electrostatic wave modes associated with the oscillations of dust dipoles around their mean positions $[44,45,46,47,48]$. We note that in oscillons the restoring force comes from the thermal pressures of electrons and ions, and the inertia is provided by the dust dipole moment. For oscillons of arbitrary wavelength and arbitrary ion gyroradius $\rho_{i}$, we use a kinetic theory in order to calculate the ion density perturbation. Thus, as before, for $\omega \ll \omega_{c i}$ the ion susceptibility $\chi_{i}$ is given by Eq. (4).

The electron density perturbation, for $b_{e}=k_{\perp}^{2} v_{t e}^{2} / \omega_{c e}^{2} \ll 1$ and $\omega \ll \omega_{c e}$, is given by $n_{e 1}=$ 

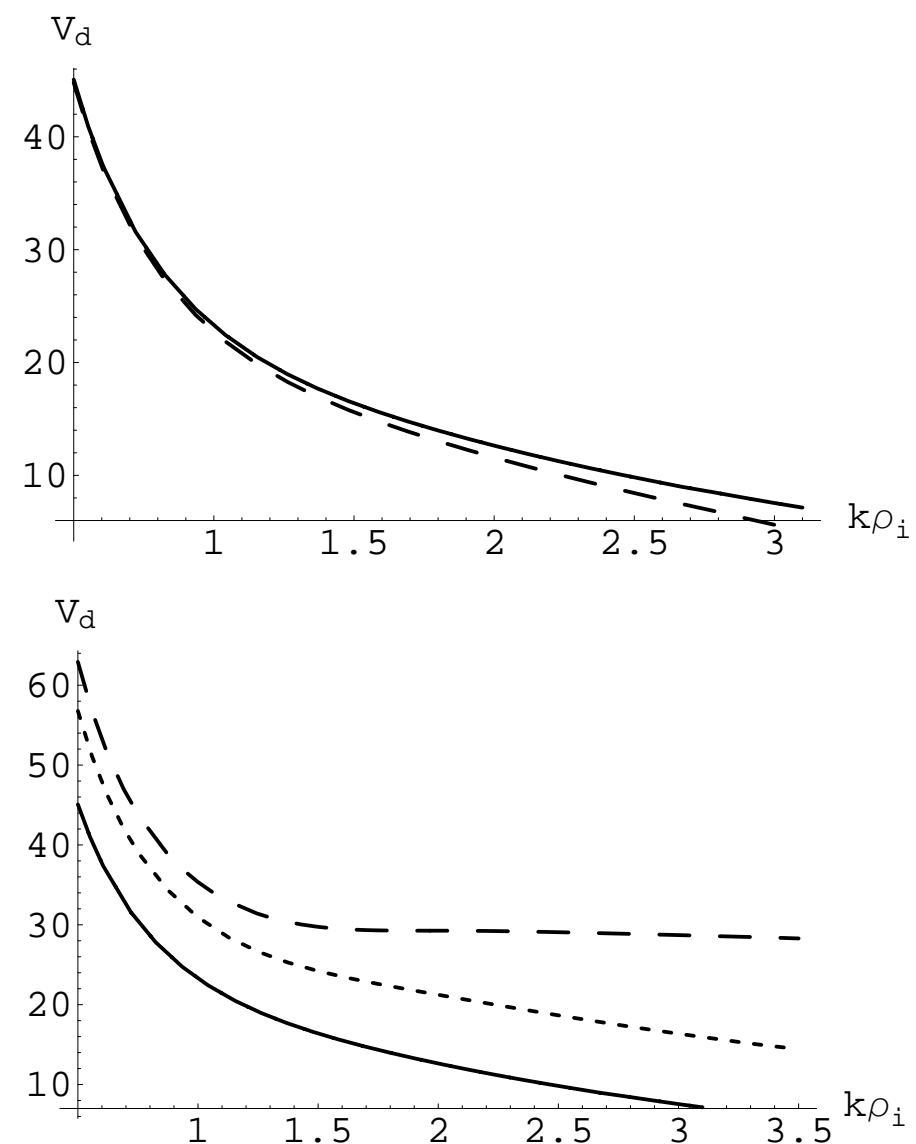

Figure 6: Showing $\Psi \equiv V_{p} / V_{d}=1$ curves in $\left(V_{d}, k \rho_{i}\right)$ space for typical laboratory dusty plasma parameters (given in the text). The upper plot, where solid (dashed) curve represents to the numerical results based on the QLCA (GHM), shows a comparison between the QLCA and the GHM. The lower plot, where $\mathcal{D}=\mathcal{D}_{R K}$, $B_{0}=10^{3} \mathrm{G}$ (solid curve), $B_{0}=5 \times 10^{3} \mathrm{G}$ (dotted curve), and $B_{0}=10^{4} \mathrm{G}$ (dashed curve), shows the effect of $B_{0}$ on $\Psi=1$ curves in $\left(V_{d}, k \rho_{i}\right)$ space.

$\left(k^{2} / 4 \pi e\right) \chi_{e} \phi$, where the electron susceptibility is

$$
\chi_{e} \simeq \frac{\omega_{p e}^{2}}{\omega_{c e}^{2}} \frac{k_{\perp}^{2}}{k^{2}}-\frac{\omega_{p e}^{2}}{\omega^{2}-\gamma_{e} k_{z}^{2} v_{t e}^{2}} \frac{k_{z}^{2}}{k^{2}}
$$

We consider $\chi_{e}$ in two limiting cases: First, $\omega \gg \sqrt{\gamma_{e}} k_{z} v_{t e}$ which reduces Eq. (19) to

$$
\chi_{e} \simeq \frac{\omega_{p e}^{2}}{\omega_{c e}^{2}} \frac{k_{\perp}^{2}}{k^{2}}
$$

and second, $\omega \ll \gamma_{e} k_{z} v_{t e}$ which reduces Eq. (19) to

$$
\chi_{e} \simeq \frac{1}{k^{2} \lambda_{D e}^{2}}
$$

where $\gamma_{e}=1$ is used.

We are interested in $\omega_{c d} \ll \omega$ so that dust grains can be treated as unmagnetized. The dust number density perturbation can, therefore, be expressed as $n_{d 1}=\left(k^{2} / 4 \pi Z_{d} e\right) \chi_{d} \phi$. For $\omega_{p d} \ll \Omega_{r}$ and $\Omega_{0} \ll \omega$ [where $\Omega_{r}=\left(4 \pi d^{2} n_{d 0} I\right)^{1 / 2}$ is the oscillon frequency, $\Omega_{0}=M / I, d$ is 
the magnitude of the dipole moment, $I$ is the moment of inertia of the dipole, and $M$ is the z-component of the dust angular momentum] the dust susceptibility $\chi_{d}$ is given by $[44,45,46$, $47,48]$

$$
\chi_{d} \simeq-\frac{\Omega_{r}^{2}}{\omega^{2}} \frac{k_{\perp}^{2}}{k^{2}} .
$$

Now, using Eqs. (4), (20)-(22) in $1+\chi_{e}+\chi_{i}+\chi_{d}=0$, the dispersion relation involving $\Psi=\omega / k V_{d}$ can be expressed as [49]

$$
\alpha_{1,2}+\frac{2 V_{d}^{2} \Lambda_{1}}{\lambda_{D i}^{2} \omega_{c i}^{2}} \Psi^{2}-\frac{\Omega_{r}^{2} k_{\perp}^{2}}{k^{4} V_{d}^{2} \Psi^{2}}=0,
$$

where $\alpha_{1}=1+\omega_{p e}^{2} k_{\perp}^{2} / \omega_{c e}^{2} k^{2}+\left(1-\Lambda_{0}\right) / k^{2} \lambda_{D i}^{2}$ corresponds to the first limiting case $\omega \gg \sqrt{\gamma_{e}} k_{z} v_{t e}$, and $\alpha_{2}=1+1 / k^{2} \lambda_{D e}^{2}+\left(1-\Lambda_{0}\right) / k^{2} \lambda_{D i}^{2}$ corresponds to the second limiting case $\omega \ll k_{z} v_{t e}$. We note that the dust particle speed $V_{d}$ in Eq. (23) is considered as a free parameter.

The Mach cones can be formed by oscillons defined by Eq. (23) if $\Psi=V_{p} / V_{d}<1$. If this condition is satisfied, the Mach cone opening angle $\theta$ is given by $\theta=\sin ^{-1} \Psi$, where $\Psi$ can be obtained from Eq. (23).

To analyze the possibility for the formation of the Mach cones associated with the waves defined by Eq. (23), we numerically analyze Eq. (23), and find $\Psi=1$ curves in $\left(V_{d}, k \rho_{i}\right)$ space for typical laboratory dusty plasma parameters: [50, 51, 52, 53, 54]: $T_{e} \simeq 10 \mathrm{eV}, T_{i} \simeq 0.2 T_{e}$, $n_{i 0} \simeq 2.5 \times 10^{9} \mathrm{~cm}^{-3}, Z_{d} \simeq 5 \times 10^{4}$, and $n_{d 0} \simeq 10^{3} \mathrm{~cm}^{-3}$. The numerical results are displayed in figures 7 and 8 . Figure 7 shows the $\Psi=1$ curves in $\left(V_{d}, k \rho_{i}\right)$ space for both $\omega \gg \sqrt{\gamma_{e}} k_{z} v_{t e}$ (upper plot) and $\omega \ll k_{z} v_{t e}$ (lower plot). The $\Psi=1$ curves in $\left(V_{d}, k \rho_{i}\right)$ space determines the critical values of the dust particle speed $V_{d}$ and the corresponding wavelength $\lambda=2 \pi / k$ for which the Mach cones are formed. The regions above the $\Psi=1$ curves correspond to $\Psi<1$, i.e. correspond to a regime for which the Mach cones are formed. It implies that for a wave of fixed wavelength as we increase $\Omega_{r}$, we need a dust particle of higher speed in order for the formation of the Mach cones in both the limits. Figure 8 depicts the variation of the Mach opening cone angle with $k \rho_{i}$ and $\Omega_{r}$ for typical laboratory dusty plasma parameters. we find that the Mach cone opening angle $\theta$ decreases with the wavelength, but increases with $\Omega_{r}$. It is observed here that the effect of $\Omega_{r}$ is stronger for $\omega \ll k_{z} v_{t e}$ (lower plot) than that for $\omega \gg \sqrt{\gamma_{e}} k_{z} v_{t e}$ (upper plot). We have also analyzed the variation of the Mach cone opening angle with $B_{0}$ for typical laboratory dusty plasma parameters. It indicates that the Mach cone opening angle $\theta=\sin ^{-1} \Psi$ decreases with the magnitude of the external field. We have found that the effect of the external magnetic field is slightly stronger for $\omega \ll k_{z} v_{t e}$ than that for $\omega \gg \sqrt{\gamma_{e}} k_{z} v_{t e}$.

\section{Discussions}

We have presented different theoretical models for the formation of Mach cones in space and laboratory dusty magnetoplasmas. We have numerically analyzed $V_{d} / V_{p}$ which is decisive for the condition $\left(V_{d} / V_{p}>1\right)$ required for the formation of Mach cones, as well as their opening angles $\left[\theta=\sin ^{-1}\left(V_{p} / V_{d}\right)\right]$. The results, which have been found from this thorough investigation, can be summarized as follows: 

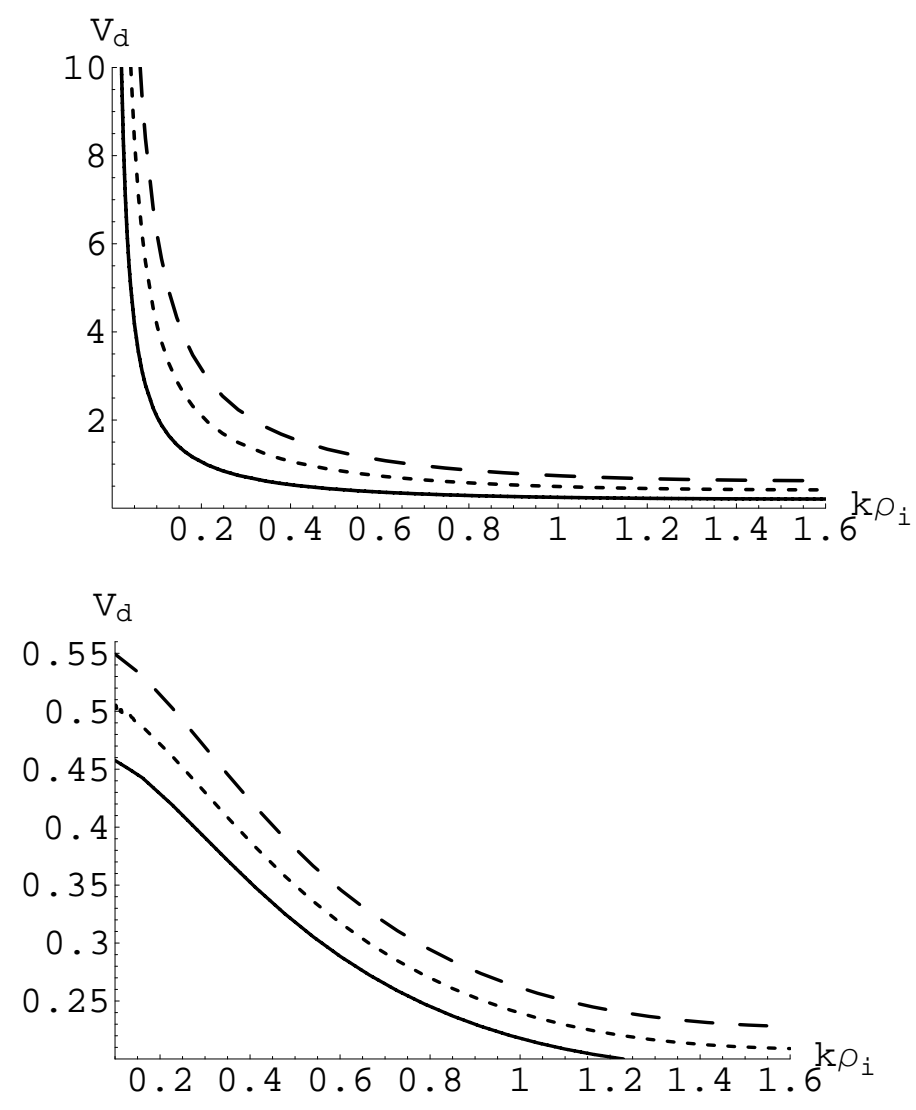

Figure 7: Showing $\Psi \equiv V_{p} / V_{d}=1$ curves in $\left(V_{d}, k \rho_{i}\right)$ space for typical laboratory dusty plasma parameters given in the text, and $B_{0}=0.01 \mathrm{~T}$. The upper plot, where $\Omega_{r}=10 \mathrm{radian} / \mathrm{s}$ (solid curve), $\Omega_{r}=20 \mathrm{radian} / \mathrm{s}(\mathrm{dotted}$ curve) and $\Omega_{r}=30 \mathrm{radian} / \mathrm{s}$ (dashed curve), corresponds to $\omega \gg \sqrt{\gamma_{e}} k_{z} v_{t e}$. The lower plot, where $\Omega_{r}=10$ radian $/ \mathrm{s}$ (solid curve), $\Omega_{r}=11 \mathrm{radian} / \mathrm{s}$ (dotted curve) and $\Omega_{r}=12 \mathrm{radian} / \mathrm{s}$ (dashed curve), corresponds to $\omega \ll k_{z} v_{t e}$.

i) We have considered short wavelength modified DAWs, long wavelength DMWs, and analyzed the condition for the formation of Mach cones associated with these waves in Saturn's dusty rings. We have found that short wavelength (in comparison with the ion gyro-radius), intermediate frequency $\left(\omega_{c d} \ll \omega \ll \omega_{c i}\right)$ modified DAWs and long wavelength $\left(k_{\perp}^{2} \rho_{i}^{2} \ll 1\right)$, ultra low-frequency $\left(\omega \ll \omega_{c d}\right)$ slow DMWs are viable candidates for the formation of Mach cones in the equatorial region of Saturn's dusty rings. The Mach cones may arise owing to the constructive interference of the modified DAWs (in the short wavelength limit) or slow DMWs (in the long wavelength limit) in dusty magnetoplasmas of Saturn's dusty rings. We expect that the NASA/ESA space probe CASSINI can make direct observations of Mach cones involving the modified DAWs and slow DMWs, which are reported in this paper. The opening angles of the corresponding Mach cones, which we have estimated herein, can be used for obtaining the dust mass density, dust charge, dust composition, and the optical depth of Saturn's dusty rings.

ii) We have investigated the possibility for the formation of the Mach cones in a laboratory dusty magnetoplasma whose constituents are weakly correlated magnetized electrons and ions, and strongly coupled unmagnetized charged dust grains. We have graphically shown the para- 

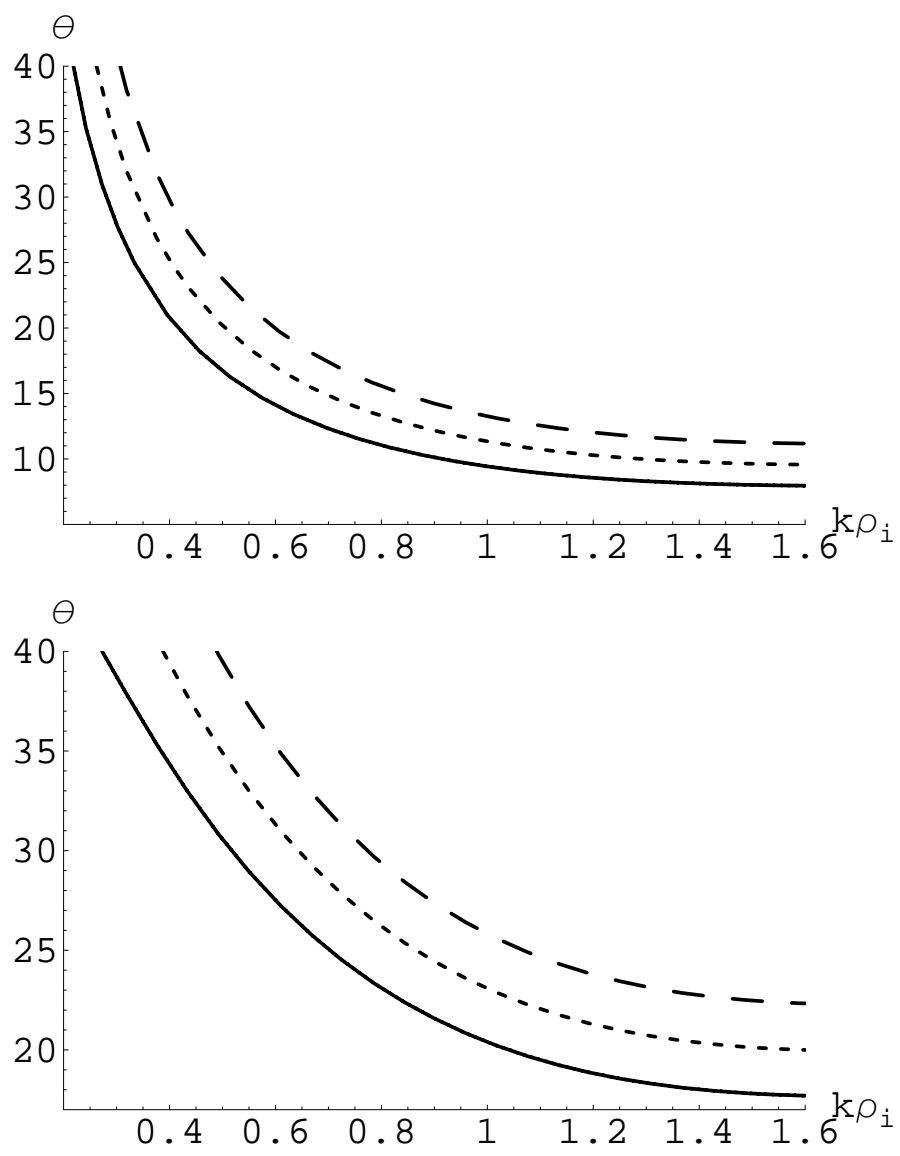

Figure 8: Showing the variation of the Mach cone opening angle with $k \rho_{i}$ and $\Omega_{r}$ for typical laboratory dusty plasma parameters given in the text, and $B_{0} \simeq 0.01 \mathrm{~T}$. The upper plot, where $V_{d}=1.5 \mathrm{~cm} / \mathrm{s}, \Omega_{r}=10 \mathrm{radian} / \mathrm{s}$ (solid curve), $\Omega_{r}=12 \mathrm{radian} / \mathrm{s}$ (dotted curve) and $\Omega_{r}=14 \mathrm{radian} / \mathrm{s}$ (dashed curve), corresponds to $\omega \gg \sqrt{\gamma_{e}} k_{z} v_{t e}$. The lower plot, where $V_{d}=0.5 \mathrm{~cm} / \mathrm{s}, \Omega_{r}=8 \mathrm{radian} / \mathrm{s}$ (solid curve), $\Omega_{r}=9 \mathrm{radian} / \mathrm{s}$ (dotted curve) and $\Omega_{r}=10$ radian/s (dashed curve), corresponds to $\omega \ll k_{z} v_{t e}$.

metric regime for which the modified DA Mach cones can be formed in a strongly cupled laboratory dusty magnetoplasma $[4,7,8,9]$. We have considered the QLCA as well as the GHM, and have found that for laboratory dusty magnetoplasma conditions the QLCA and the GHM do not have any significant discrepancies, and for $k \rho_{i} \leq 1$ both approaches give exactly the same results. We have also examined the effect of the external magnetic field on the critical values of the dust particle speed $V_{d}$ and the wavelength of the waves for which the Mach cones may be formed in a strongly coupled dusty magnetoplasma. We found that as we increase the magnitude of the external magnetic field, for waves of fixed wavelength we need a dust particle of higher speed in order for the creation of Mach cones.

iii) We have also investigated the possibility for the formation of the Mach cones in a dusty magnetoplasma whose constituents are magnetized electrons and ions as well as unmagnetized negatively charged elongated (rod-like) dust grains. We have graphically shown the parametric regimes for which the oscillonc Mach cones can be formed in laboratory dusty magnetoplasmas $[50,51,53,54]$. We have found that for waves of fixed wavelength, as we increase the magnitude 
of $\Omega_{r}$, we need a dust particle of higher speed in order for the creation of the Mach cones. We also observed that the Mach cone opening angle $\theta$ decreases with the wavelength and with the magnitude of the external magnetic field strength, but increases with the dust dipole oscillon frequency $\left(\Omega_{r}\right)$. We finally propose to conduct laboratory experiments for verifying the theoretical prediction that we made herein.

Acknowledgments. This work was partially supported by the Regular Associateship Scheme of the Abdus Salam International Centre for Theoretical Physics, Trieste, Italy, as well as by the Swedish International Development Cooperation Agency (SIDA). 


\section{References}

[1] H. W. Liepman and A. Roshko, Elements of Gas Dynamics (Wiely, New York, 1957).

[2] J. Bond, K. Watson, and J. Welch, Atomic Theory of Gas Dynamics (Addison-Wesley, Reading, 1965).

[3] G. Crapper, Introduction to Water Waves (Chichester, Harwood, 1984).

[4] D. Samsonov et al., Phys. Rev. Lett. 83, 3649 (1999).

[5] P. Gumbsch and H. Gao, Science 283, 965 (1999).

[6] D. E. Dubin, Phys. Plasmas 7, 3895 (2000).

[7] A. Melzer et al., Phys. Rev. E 62, 4162 (2000).

[8] V. Nosenko et al., Phys. Rev. Lett. 88, 135001 (2002).

[9] H. Thomas et al., Plasma Phys. Rep. 29, 895 (2003).

[10] N. Cheng, Z. Zhu, C. Cheng, and M. Toksöz, Geophysical Prospecting 42, 303 (1994).

[11] B. A. Smith et al., Science 212, 163 (1981).

[12] J. R. Hill and D. A. Mendis, Moon and Planets 24, 431 (1981).

[13] O. Havnes et al., J. Geophys. Res. 100, 1731 (1995).

[14] O. Havnes et al., J. Vac. Sci. Technol. A 14, 525 (1996).

[15] A. Brattli, O. Havnes, and F. Melandsø, Phys. Plasmas 9, 985 (2002).

[16] N. N. Rao, P. K. Shukla, and M. Y. Yu, Planet. Space Sci. 38, 543 (1990).

[17] D. A. Mendis, H. L. F. Houpis, and J. R. Hill, J. Geophys. Res. 87, 3449 (1982).

[18] P. K. Shukla and A. A. Mamun, Phys. Lett. A 315, 451 (2003).

[19] D. A. Mendis and M. Rosenberg, Annu. Rev. Astron. Astrophys. 32, 419 (1994).

[20] D. A. Mendis, Plasma Sources Sci. Technol. 11, A219 (2002).

[21] J. E. Howard, M. Horányi, and G. R. Stewart, Phys. Rev. Lett. 83, 3993 (1999).

[22] M. Horányi, Phys. Plasmas 7, 3847 (2000).

[23] F. Verheest, Waves in Dusty Space Plasmas (Kluwer Academic Publ., Dordrecht, 2000).

[24] P. K. Shukla and A. A. Mamun, Introduction to Dusty Plasma Physics (IoP Publ. Ltd., Bristol, 2002).

[25] A. A. Mamun, P. K. Shukla, and R. Bingham, JETP Lett. 77, 541 (2003).

[26] P. K. Shukla, A. A. Mamun, and R. Bingham, JETP Lett. 78, 99 (2003).

[27] A. A. Mamun, P. K. Shukla, and G. Morfill, Phys. Rev. Lett. 92, 095005 (2004). 
[28] A. A. Mamun and P. K. Shukla, Geophys. Res. Lett. 31, L06808 [doi: 10.1029/2003GL018684] (2004).

[29] P. K. Shukla, A. A. Mamun, and R. Bingham, Physica Scripta 69, 48 (2004).

[30] M. Horányi, Annu. Rev. Astron. Astrophys. 34, 383 (1996).

[31] V. E. Fortov et al., JETP 87, 1087 (1998).

[32] J. E. Howard and M. Horányi, Geophys. Res. Lett. 28, 1907 (2001).

[33] M. Brambilla, Kinetic Theory of Plasma Waves: Homogeneous Plasmas (Clarendon Press, Oxford, 1998).

[34] P. K. Shukla and L. Stenflo, J. Plasma Phys. 64, 125 (2000).

[35] P. K. Shukla, Phys. Rev. E, 61, 7249 (2000).

[36] S. A. Khrapak and G. E. Morfill, Phys. Plasmas, 8, 2629 (2001).

[37] V. E. Fortov et al., New J. Phys. 5, 102 (2003).

[38] M. Rosenberg and G. Kalman, Phys. Rev. E. 56, 7166 (1997).

[39] P. K. Kaw and A. Sen, Phys. Plasmas 5, 3552 (1998).

[40] P. K. Shukla, M. Torney, R. Bingham, and G. E. Morfill, Phys. Scripta 67, 350 (2003).

[41] S. Ichimaru, H. Iyetomi, and S. Tanaka, Phys. Rep. 149, 91 (1987).

[42] W. L. Slattery, G. D. Doolen, and H. E. DeWitt, Phys. Rev. A 21, 2087 (1980).

[43] D. Winske and M. E. Jones, IEEE. Trans. Plasma Sci. 22, 454 (1994).

[44] J. Mahmoodi, P. K. Shukla, N. L. Tsintsadze, and D. D. Tskhakaya, Phys. Rev. Lett. 84, $2626(2000)$.

[45] D. D. Tskhakaya and P. K. Shukla, Phys. Lett A 286, 277 (2001).

[46] P. K. Shukla and D. D. Tskhakaya, Phys. Plasmas 8, 661 (2001).

[47] P. K. Shukla, Phys. Lett A 289, 89 (2001).

[48] M. Salimullah, P. K. Shukla, I. Sandberg, and G. E. Morfill, New J. Phys. 5, 40 (2003).

[49] P. K. Shukla, A. A. Mamun, B. Eliasson, and G. E. Morfill, Phys. Plasmas 11 (8), 0000 (2004).

[50] U. Mohideen, H. U. Rahman, M. A. Smith, M. Rosenberg, and D. A. Mendis, Phys. Rev. Lett. 81, 349 (1998).

[51] V. I. Molotkov, A. P. Nefedov, M. Yu. Pustyl'nik et al., JETP Lett. 71, 102 (2000).

[52] V. E. Fortov, A. P. Nefedov, V. M. Torchinsky et al., Phys. Lett. A 229, 317 (1997).

[53] D. Samsonov, J. Goree, Z. W. Ma, A. Bhattacharjee, H. M. Thomas, and G. E. Morfill, Phys. Rev. Lett. 61, 5557 (2000).

[54] D. Samsonov, J. Goree, H. M. Thomas, and G. E. Morfill, Phys. Rev. E 61, 5557 (2000). 